\title{
A Logistic Formula in Biology and Its Application to Deaths by the Third Wave of COVID-19 in Japan
}

\author{
Akira Kokado* \\ Kobe Internationa Univerlsity, Kobe 658-0032, Japan \\ and \\ Takesi Saito $^{\dagger}$ \\ Department of Physics, Kwansei Gakuin University, Sanda 669-1337, Japan
}

\begin{abstract}
A logistic formulation in biology is applied to analyze deaths by the third wave of COVID19 in Japan.

\section{Introduction}

In previous papers [1], [2] we have proposed a logistic formulation of infection. The logistic formula is useful in the population problem in biology. It is closely related to the SIR model [3] in the theory of infection. which is powerful to analyze an epidemic about how it spreads and how it ends [4-11]. The SIR model is composed of three equations for S, I and R, where they are numbers for susceptibles, infectives and removed, respectively. Our logistic formula has been driven approximately from this SIR model. This approximate formula, however, has a simple form, so that it is very useful to discuss an epidemic.

In this paper, our policy is to use mainly data of deaths by COVID-19 in Japan, but not so often of cases.

In Sec. 2, we review the derivation of this logistic formula and construct basic equations used later. In Sec. 3, our logistic formula is applied to the $3^{\text {rd }}$ wave of COVID-19 in Japan. The final section is devoted to concluding remarks. We attach Appendix for error estimations.
\end{abstract}

\section{The logistic formula}

In previous works [1], [2] we found a logistic formula, which is an approximate one of the SIR model [3] in the theory of infection. Let us summarize the formula briefly. The third equation of the SIR model is given by

$$
\frac{d R}{d t^{\prime}}=I\left(t^{\prime}\right)=1-R-\exp (-\alpha R), \quad t^{\prime}=c t,
$$

*kokado@kobe-kiu.ac.jp

†tsaito@k7.dion.ne.jp

NOTE: This preprint reports new research that has not been certified by peer review and should not be used to guide clinical practice. 
where $R$ is the removed number, $I\left(t^{\prime}\right)$ the infectious number, $\alpha$ the basic reproduction number, $c$ the removed ratio and $t$ the true time. There is another function $S$ in the SIR model, which stands for the susceptible number. Three functions $S, I$ and $R$ are normalized as $S+I+R=1$.

Let us expand the exponential factor as, with $x=\alpha R$, in the second order,

$$
\exp (-x)=1-x+\frac{1}{2} x^{2}
$$

Here we have assumed $x^{2} / 2<1$. In this approximation we have

$$
\frac{d R}{d t^{\prime}}=I\left(t^{\prime}\right)=1-R-\left(1-\alpha R+\frac{1}{2} \alpha^{2} R^{2}\right)=A(B-R) R
$$

with $A=\alpha^{2} / 2, B=2(\alpha-1) / \alpha^{2}$. This equation is a type of that in the logistic growth curve in Biology, easily solved as

$$
R(t)=\frac{R(\infty)}{1+\exp (-a c(t-T))} \cdot \quad a=\alpha-1
$$

where $A B=\alpha-1=a$ and $B=R(\infty)=2(\alpha-1) / \alpha^{2}=2 R(T), T$ being the peak day of infection. Inserting this into Eq.(2.1) we get:

$$
I(t)=\frac{a R(\infty)}{2(1+\cosh a c(t-T))},
$$

We make use of data [12] of deaths rather than cases. It is our policy not to use data of cases. Define the mortality ratio $\lambda$ by

$$
\lambda=\frac{D(t)}{R(t)}
$$

where $D(t)$ is the accumulated number of deaths. For the population $N$, the total number of deaths at $\mathrm{t}$ is given by

$$
N D(t)=\lambda N R(t)=\frac{d}{1+\exp (-z)}, \quad z=a c(t-T)
$$

where $d=N D(\infty)=\lambda N R(\infty)$ stands for the final total number of deaths. Note that from Eq.(2.7) we have a theorem, $N D(T)=d / 2$, which means the total number of deaths at the peak is just a half of the final ones.

Let us rewrite Eq.(2.7) in a form as

$$
-z=a c(T-t)=\ln F(t), \quad F=\frac{d}{N D(t)}-1 .
$$

Accordingly, for different times $t_{1}, t_{2}$ and $t_{3}$ we have equations

$$
\begin{aligned}
& a c\left(t_{2}-t_{1}\right)=\ln \frac{F\left(t_{1}\right)}{F\left(t_{2}\right)}, \\
& a c\left(t_{3}-t_{2}\right)=\ln \frac{F\left(t_{2}\right)}{F\left(t_{3}\right)} .
\end{aligned}
$$

When time differences in Eqs. (2.9) and (2.10) are equal, we get a useful equation for $d$

$$
F\left(t_{1}\right) F\left(t_{3}\right)=F\left(t_{2}\right)^{2}
$$




\section{Application to the third wave of COVID-19}

Our logistic formula is applied to the $3^{r d}$ wave of COVID-19 in Japan.

Table 1: Date and the number of deaths in the $3^{\text {rd }}$ wave

\begin{tabular}{cc}
$\mathrm{t}$ & $\mathrm{N} \mathrm{D}(\mathrm{t})$ \\
\hline$t_{1}=$ Dec. 17 & $n_{1}=1150.143 \pm 95.106$ \\
$t_{2}=$ Dec. 31 & $n_{2}=1826.714 \pm 106.463$ \\
$t_{3}=$ Jan. 14 & $n_{3}=2673 \pm 105.347$
\end{tabular}

$N D(t)$ is the accumulated number of deaths in the $3^{r d}$ wave at $t$ in Japan, which is an average of deaths for 7 days in a middle at each $t$ with standard deviations, where $t$ is the date starting from Oct. 11. We have subtracted the accumulated number of deaths, 1628 , in the $1^{\text {st }}$ and $2^{\text {nd }}$ waves, from that in the $1^{\text {st }}, 2^{\text {nd }}$ and $3^{\text {rd }}$ waves.

A use is made of data of deaths [12]. Substituting data on the Table 1 into Eq.(2.11), we get the equation for $d$

$$
\left(\frac{d}{1150.143}-1\right)\left(\frac{d}{2673}-1\right)=\left(\frac{d}{1826.714}-1\right)^{2} .
$$

We find a solution of this equation to be $d=5810.410$. According to the theorem $N D(T)=$ $d / 2$, the number of deaths at the peak $t=T$ is $d / 2=2905.205$. The result of $d=5810.410$ is substituted into Eq.(2.9), then it follows that

$$
14 a c=\ln \frac{F\left(t_{1}\right)}{F\left(t_{2}\right)}=\ln \frac{4.0519}{2.1808}=0.6195,
$$

hence

$$
a c=0.04425
$$

and

$$
a c\left(T-t_{3}\right)=\ln F\left(t_{3}\right)=\ln 1.1737=0.1602 .
$$

The last equation means $T-t_{3}=3.62 \simeq 4$, hence $T=$ Jan. $14+4=$ Jan.18.

Error estimations for $d=5810.410$ and $T=\operatorname{Jan} .18$ (=99) can be seen from Appendix, i.e.,

$$
\frac{\delta d}{d}=0.0331
$$

which states $\delta d=192.3$ and $\delta T=-0.2$.

To sum up we have

$$
\begin{gathered}
\frac{d}{2}=2905 \pm 96.15 \\
T=\text { Jan. } 18 \pm 1
\end{gathered}
$$

In order to fix a, we make use of $c=0.041$, which is frequently quoted value. Since $a c=0.044$, we have $a=1.07$, that is, $\alpha=1+a=2.07$. Once having $\alpha=2.07$, we can draw curves of $S, I$ and $R$ by means of Excel in Fig. 1. From this we find $t^{\prime}=4.86$ at the peak of $I$, so that from the formula $t^{\prime}=c t$ we get $c=t^{\prime} / T=4.86 / 99=0.049$. This value $c=0.049$ is slightely different from $c=0.041$, that is, its error is $16 \%$. About the error we have discussed in a previous paper [1] that the error of our logistic model against the SIR model is about 15\%. Therefore, this difference may be allowed. 


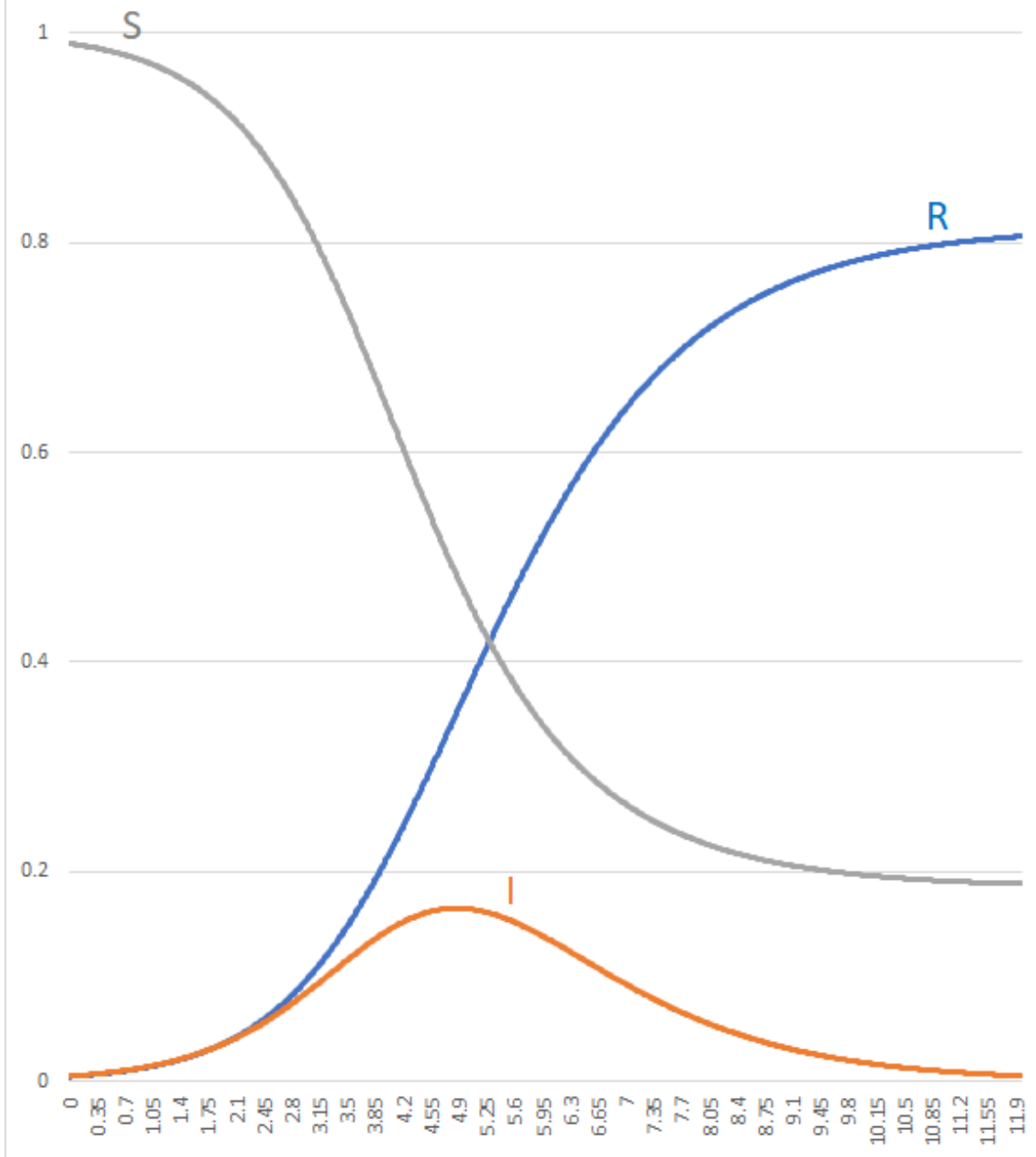

Figure 1: Graph of $S, I$ and $R$ for $\alpha=2.07$ and $c=0.041$

\section{Concluding remarks}

We found that the third wave began from Oct. 11 and will peak at around Jan. $18 \pm 1,2021$ with total deaths $2905 \pm 96$. The total number of deaths for the $1^{\text {st }}, 2^{\text {nd }}$ and $3^{\text {rd }}$ waves on the peak is $2905 \pm 96+1628=4593 \pm 96$. This should be compared with the observed value 4547. The basic reproduction number of the third wave is $\alpha=2.07$ with the removed ratio $c=0.041$. Curves of $S, I$ and $R$ are given in Fig. 1

Let us discuss the population related with infectives. Japanese population is divided into two groups, one is self-isolated and the other is not self-isolated. We can remove the self-isolated group from the infection route, because it is irrelevant to the infection. The nonisolated group is, therefore, relevant to the infection. We put such a population as $N$. From Fig. 1, one can see that

$$
R(\text { Jan. } 18)=R\left(t^{\prime}=4.86\right)=0.3520
$$

$N R(\operatorname{Jan} .18)=$ accumulated numbers of (deaths + discharged $)$

$$
=2919+174582=177501=N \times 0.3520
$$


yield $N=504264$. Therefore, we conclude that the non-isolated population $N$ in the $3^{r d}$ wave is about 504,000. We can then estimate the number of infectives on Jan. 18 by formulas

$$
I\left(\text { Jan.18) }=I\left(t^{\prime}=4.86\right)=0.1654\right.
$$

and

$$
N I(\text { Jan. } 18)=504,000 \times 0.1654=83362 \simeq 83,000 .
$$

The present number of infectives on Jan. 18 in Japan is about 74,000 and seems to be slightly lower than the calculated value 83,000 .

\section{Acknowledgement}

We would like to express our deep gratitude to K. Shigemoto for many valuable discussions and big supports.

\section{Appendix Error estimations}

The total death $d$ is given by

$$
d=\frac{B}{A}=\frac{n_{2}^{2}\left(n_{1}+n_{2}\right)-2 n_{1} n_{2} n_{3}}{n^{2}-n_{1} n_{3}} .
$$

The relative error of $d$ is then derived by

$$
\frac{\delta d}{d}=\frac{\delta B}{B}-\frac{\delta A}{A}
$$

where

$$
\begin{gathered}
\delta A=2 n_{2}^{2} \frac{\delta n_{2}}{n_{2}}-n_{1} n_{3}\left(\frac{\delta n_{1}}{n_{1}}+\frac{\delta n_{3}}{n_{3}}\right) \\
\delta B=\frac{\delta n_{1}}{n_{1}}\left(n_{1} n_{2}^{2}-2 n_{1} n_{2} n_{3}\right)+\frac{\delta n_{2}}{n_{2}}\left[2 n_{2}^{2}\left(n_{1}+n_{3}\right)-2 n_{1} n_{2} n_{3}\right]+\frac{\delta n_{3}}{n_{3}}\left[n_{2}^{2} n_{3}-2 n_{1} n_{2} n_{3}\right]
\end{gathered}
$$

Substituting $\frac{\delta n_{1}}{n_{1}}=0.0827, \frac{\delta n_{2}}{n_{2}}=0.0583, \frac{\left.\delta n_{3}\right)}{n_{3}}=0.0394$ into above, we get

$$
\frac{\delta d}{d}=0.0331
$$

In the same way, from the equation for $a c$

$$
14 a c=\ln \frac{F_{1}}{F_{2}},
$$

we have

$$
14 \delta(a c)=\frac{\delta F_{1}}{F_{1}}-\frac{\delta F_{2}}{F_{2}}=\frac{\delta d}{d}\left(\frac{1+F_{1}}{F_{1}}-\frac{1+F_{2}}{F_{2}}\right)-\frac{1+F_{1}}{F_{1}} \frac{\delta n_{1}}{n_{1}}+\frac{1+F_{2}}{F_{2}} \frac{\delta n_{2}}{n_{2}},
$$

so that

$$
\delta(a c)=-0.00079 \simeq-0.001 .
$$

Finally, the equation for the peak day $T, a c\left(T-t_{3}\right)=\ln F_{3}$, yields

$$
\delta T=-0.1998 \simeq-0.2 .
$$




\section{References}

[1] T. Saito and K. Shigemoto, "A Logistic Curve in the SIR Model and Its Application to Deaths by COVID-19 in Japan ", [medRxiv 10.1101/2020.06.25.20139865](2020).

[2] T. Saito An Application of Logistic Formula to Deaths by COVID-19 in Japan", [medRxiv 10.1101/2020. 09.15.20195081](2020).

[3] W. O. Kermack and A.G. McKendrick, "A Contribution to the Mathematical Theory of Epidemics", Proceedings of the Royal Society A115 , 700-721 (1927)..

[4] W. D. Murray, "Epidemic models and the dynamics of infectious diseases", Mathematical Biology 42, 610-650 (1993).

[5] H. Hethcote, "The Mathematics of Infectious Diseases", SIAM Review 42, 599-653 (2000).

[6] M.J. Keeling and L. Danon, "Mathematical modelling of infectious diseases ", British Medical Bulletin 92, 33-42 (2009).

[7] S. Pathak, A. Maiti and G.P. Samanta, "Rich dynamics of an SIR epidemic model", Nonlinear Analysis: Modelling and Control 15, 71-81 (2010).

[8] T. Harko, F.S.N. Lobo, M.K. Mak, "Exact analytical solutions of the SusceptibleInfected-Recovered (SIR) epidemic model and of the SIR model with equal death and birth rates", Applied Mathematics and Computation 236, 184194 (2014).

[9] J.C. Miller, "Mathematical models of SIR disease spread with combined non-sexual and sexual transmission routes", Infectious Disease Modelling 2, 3555 (2017).

[10] R. Sameni, "Mathematical Modeling of Epidemic Diseases; A Case Study of the COVID19 Coronavirus", [arXiv:2003.11371 [q-bio.PE]](2017).

[11] P-F. Verhulst, "Notice sur la loi que la population poursuit dans son accroissement", Correspondance Mathmatique et Physique 10, 113-121 (1838).

[12] Data published from the Ministry of Health, Labor and Welfare of Japan. See for example, "Summary of the New Coronavirus Infection "(in Japanese), https://hazard.yahoo.co.jp/article/20200207; "Status of the Domestic New Coronavirus Infection" (in Japanese), https://toyokeizai.net/sp/visual/tko/covid19/. 